\title{
INCIDENTAL CAPTURES OF LEPIDOPTERA AT PLANO, TEXAS.
}

BY E. S. TUCKer, BUREAU OF ENTOMOlogy, U. S. DEPT. OF AGRICUlture.

IN connection with my study of the green bug at Plano, Collin county, Texas, from the first week of May to the end of December, 1907, a considerable number of insects in all of the principal orders were personally collected, and the results obtained from the identifications of the Lepidoptera thus taken are herewith presented.

Only a few butterflies were captured, since opportunities for collecting Lepidoptera especially were limited mostly to night work, which, however, proved to be an enjoyable diversion. At such times, the light of one or two ordinary lanterns shining against the breeding tent was employed with good success in the attraction of nocturnal flyers, and on this account, the records of night collecting predominate in my list. The locality in which the collecting was done is fairly typical of the blackbelt prairie lands of northern Texas, where much of the country is under cultivation.

Most of the specimens were determined at the U. S. entomological laboratory in Dallas, Texas, with the assistance of Mr. F. C. Pratt, and acknowledgments are also due Dr. H. G. Dyar and Mr. A. Busck of Washington, D. C., through the courtesy of Dr. L. O. Howard, for valuable aid. For a few other determinations, credit is given in the particular cases to Dr. W. G. Dietz and Mr. W. D. Kearfott. Dr. Dyar's catalogue has been followed as a guide for the nomenclature and arrangement of my list. Permission to publish this list has been kindly granted by Prof. C. E. Sanborn, who, during the time of collection, directed my experimental work.

Summary of Families and Species.

$\begin{array}{ll}\text { Pieridæ } & 2 \\ \text { Nymphalidæ } & 5 \\ \text { Lymnædidæ } & 1 \\ \text { Lycænidæ } & \\ \text { Hesperiidæ } & 3 \\ \text { Sphingidæ } & 4 \\ \text { Saturniidæ } & 1 \\ \text { Ceratocampidæ } & 1 \\ \text { Syntomidæ } & \end{array}$

$\begin{array}{lll}2 & \text { Lithosiidæ } & \\ 5 & \text { Arctiidæ } & 5 \\ 1 & \text { Noctuidæ } & 3 \\ 1 & \text { Notodontidæ } & \\ 3 & \text { Lasiocampidæ } & \\ 4 & \text { Geometridæ } & 12 \\ 1 & \text { Nolidæ } & \\ 1 & \text { Psychidæ } \\ 2 & \text { Cochlidiidæ }\end{array}$

1
5
31
3
1
12
1
1
1

$\begin{array}{lr}\text { Megalopygidæ } & 1 \\ \text { Pyralidæ } & 34 \\ \text { Pterophoridæ } & 2 \\ \text { Tortricidæ } & 6 \\ \text { Yponomeutidæ } & 2 \\ \text { Gelechiidæ } & 1 \\ \text { Blastobasidæ } & 1 \\ \text { Elachistidæ } & 2 \\ \text { Tineidæ } & 5\end{array}$

Total number of species, 130 . 


\section{Family PIERID正.}

Pontia rapæ L. July in cabbage patch, all stages.

Pyrisita mexicana Boisd. July; July in cabbage patch.

\section{Family NYMPHALIDÆ.}

Euptoieta claudia Cram. August.

Mestra amymone Men. September.

Polygonia interrogationis Fab., var. umbrosa Lint. August.

Basilarchia archippus Cram. July.

Chlorippe celtis Bois. \& Le C. July.

Family LYMNADIDA.

Anosia berenice Cram. August.

Family LYCAENIDA.

Uranotes melinus Hbn. July.

Family HESPERIIDÆ.

Ancyloxypha numitor Fab. July.

Hylephila campestris Boisd. August.

Hesperia tessellata Sc. November.

\section{Family SPHINGIDA.}

Hemaris diffinis Boisd., var. axillaris G. \& R. July. Pholus pandorus Hbn. 'May at night taken at trap light.

Phlegethontius quinquemaculata Haw. September twilight. sexta Joh. July twilight.

Family SATURNIIDA.

Automeris io Fab. August, freshly emerged under bridge.

Family CERATOCAMPIDA.

Adelocephala bicolor Haw. August at night, taken at trap light. 
Family SYNTOMIDÆ.

Scepsis fulvicollis Hbn. July and August at night taken at trap light.

Ctenucha venosa Wk. June twilight; July; November.

\section{Family LITHOSIIDA.}

Ozonadia unifascia G. \& R. August at night taken at trap light.

\section{Family ARCTIIDÆ.}

Eubaphe aurantiaca Hbn. July at night taken at trap light; October.

Eurythra phasma Harv. August at night taken at trap light.

Estigmene acræa Dru. August at night taken at trap light.

Diacrisia virginica Fab. August at night taken at trap light.

Apantesis nais Dru. September at night taken at trap light.

\section{Family NOCTUIDÆ.}

Cyathisa percara Morr. August at night taken at trap light.

Platysenta videns Gn. August and September at night taken at trap light.

Caradrina exigua Hbn. July and September at night taken at trap light. spilomela Wk. September at night taken at trap light.

Prodenia ornithogalli Gn. July and August at night taken at trap light.

Heliophila unipuncta Haw. November; November twilight.

Grotella septempunctata Haw. September.

Heliothis obsoleta Fab., (= armiger Hbn.). July and August at night taken at trap light; July to October, worms in corn ears and on fall sprouts; October, in cotton bolls.

Schinia chrysellus Grt. September at night taken at trap light. jaguarina Gn. August at night taken at trap light.

marginata Haw. August and September at night taken at trap light.

Plusiodonta compressipalpis Gn. August at night taken at trap light.

Autographa ou Gn. May at night taken at trap light.

brassicæ Ril. August at night taken at trap light.

falcigera Kirb. November.

Pterætholix bullula Grt. September and October at night taken at trap light. Alabama argillacea Hbn. September at night taken at trap light; October. Xanthoptera nigrofimbria Gn. July. 
Metoponia obtusa H.-S. May, August and September, all at night taken at trap light.

Tarache biplaga Gn. July at night taken at trap light. erastrioides Gn. May, dusk in oat field. candefacta $\mathrm{Hbn}$. July; August at night taken at trap light.

Fruva fasciatella Grt. July and August at night taken at trap light. obsoleta Grt. September at night taken at trap light.

Spragueia leo Gn. August at night taken at trap light. dama Gn. July at night taken at trap light.

Hypsoropha hormos Hbn. July and August at night taken at trap light.

Drasteria erechtea Cram. July and September at night taken at trap light;

October; October in oat field; November; November at night taken at trap light.

Melipotis jacunda Hbn. July; September at night taken at trap light.

Homoptera lunata Dru. September.

Salia interpuncta Grt. July at night taken at trap light.

Family NOTODONTIDÆ.

Hippia packardii Morr. August at night taken at trap light.

Litodonta hydromeli Harv. August at night taken at trap light.

Schizura unicornis S. \& A, September.

\section{Family LASIOCAMPIDE.}

Heteropacha rileyana Harv. July and August at night taken at trap light.

\section{Family GEOMETRIDÆ.}

Hydriomena latirupta Wk. November.

Gypsochroa sitellata Gn. September at night taken at trap light.

Hæmatopsis grataria Fab. July in corn field; September at night taken at trap light.

Cosymbia serrulata Pack. September at night taken at trap light.

Eois ossularia Hbn. July to September at night taken at trap light.

Chlorochlamys phyllinaria Zell. May at dusk in oat field; May to September at night taken at trap light.

Fernaldella fimetaria G. \&. R. July to September at night taken at trap light. Mellilla inextricata Wk. August and September at night taken at trap light. 
Sciagraphia californiaria Pack. August at night taken at trap light.

Macaria infimata Gn. August at night taken at trap light.

s-signata Pack. August at night taken at trap light.

Tornos scolopacinarius Gn. August at night taken at trap light.

Family NOLIDAE.

Celama sorghiella Ril. August at night taken at trap light.

Family PSYCHIDA.

Eurycyttarus carbonaria Pack. November.

Family COCHLIDIID必.

Euchlea chloris H.-S. August at night taken at trap light.

Family MEGALOPYGID $\approx$.

Lagoa crispata Pack. August at night taken at trap light.

Family PYRALIDA.

Symphysa reniculalis Zell. August at night taken at trap light.

Hymenia perspectalis Hbn. August and September at night taken at trap light.

Conchylodes platinalis Gn. September at night taken at trap light.

Hellula undalis Fab. September.

Nomophila noctuella D. \& S. September at night taken at trap light.

Loxostege coloradensis G. \& R. August at night taken at trap light.

mancalis Led. August and September at night taken at trap light.

similalis Gn. July, worms and moths in alfalfa field; July and

September at night taken at trap light.

macluræ Ril. August at night taken at trap light.

Phlyctænia ferrugalis Hbn. September at night taken at trap light; October twilight; November.

Pyrausta illibalis Hbn. September at night taken at trap light.

laticlavia G. \& R. July; August at night taken at trap light.

Lineodes integra Zell. July to September at night taken at trap light.

Elophila fulicalis Clem. July to October at night taken at trap light.

Pyralis farinalis L. September; December.

Omphalocera dentosa Grt. August at night taken at trap light. 
Patissa xantholeucalis Gn. August and September at night taken at trap light. Crambus vulgivagellus Clem. October twilight.

teterrellus Zinck. August and September at night taken at trap light; October twilight.

mutabilis Clem. May at dusk in oat field; August and September at night taken at trap light.

Crambus hemiochrellus Zell. August and September at night taken at trap light.

Argyria nivalis Dru. September at night taken at trap light.

Diatræa differentialis Fern. September at night taken at trap light.

Galleria mellonella L. May, female moth disturbed from hiding-place in covered mess wagon.

Benta melanogrammos Zell. August at night taken at trap light.

Tacoma feriella Hulst. August and September at night taken at trap light.

Tlascala reductella Wk. August at night taken at trap light.

Elasmopalpus lignosellus Zell. July to September at night taken at trap light; October twilight.

Euzophera semifuneralis Wk. September at night taken at trap light.

Canarsia ulmiarrosorella Clem. July at night taken at trap light.

Honora mellinella Grt. September at night taken at trap light.

Homœosoma mucidellum Rag. September at night taken at trap light.

Saluria tetradella Zell. July and August at night taken at trap light.

Tampa dimediatella Rag. August and September at night taken at trap light.

Family P'TEROPHORIDA.

Pterophorus paleaceus Zell. August at night taken at trap light.

inquinatus Zell. July to October at night taken at trap light.

Family TORTRICIDE.

Eucosma circulana Hbn. May at night taken at trap light.

comatulana Zell. (Det. W. D. Kearfott.) September at night taken at trap light.

strenuana Wk. August at night taken at trap light.

Thiodia spiculana Zell. (Det. W. G. Dietz.) September at night taken at trap light.

Epinotia crispana Clem. (Det. W. G. Dietz.) July at night taken at trap light. 
Platynota nigrocervina Walsm. (Det. W. G. Dietz.) July to September at night taken at trap light.

Family YPONOMEUTID瓜.

Atteva punctella C. \& S., (= aurea Fitch). August at night taken at trap light. Plutella maculipennis Curt. May at dusk in oat field.

Family GELECHIIDA.

Gelechia discoocellella Cham. August and September at night taken at trap light.

\section{Family BLASTOBASIDÆE.}

Dryope ochreella Clem. (Det. W. G. Dietz.) October at night taken at trap light.

Family ELACHISTIDA.

Coleophora fuscostrigella Clem. (Det. W. G. Dietz.) October at night taken at trap light.

Scythris impositella Zell. November.

Family TINEID $\mathrm{E}$.

Tinea misella Zell. (Det. W. G. Dietz.) December twilight, in window.

Setomorpha rutella Zell., ( $+=$ operosella Zell.; $\sigma^{\nearrow}=$ in amœenella Zell.). August and September at night taken at trap light.

Acrolophus cervinus Walsm. May at night taken at trap light.

Anaphora popeanella Clem. July; July and August at night taken at trap light.

Ortholophus variabilis Walsm. July, resting under edge of clapboard on house, and at night taken at trap light. 

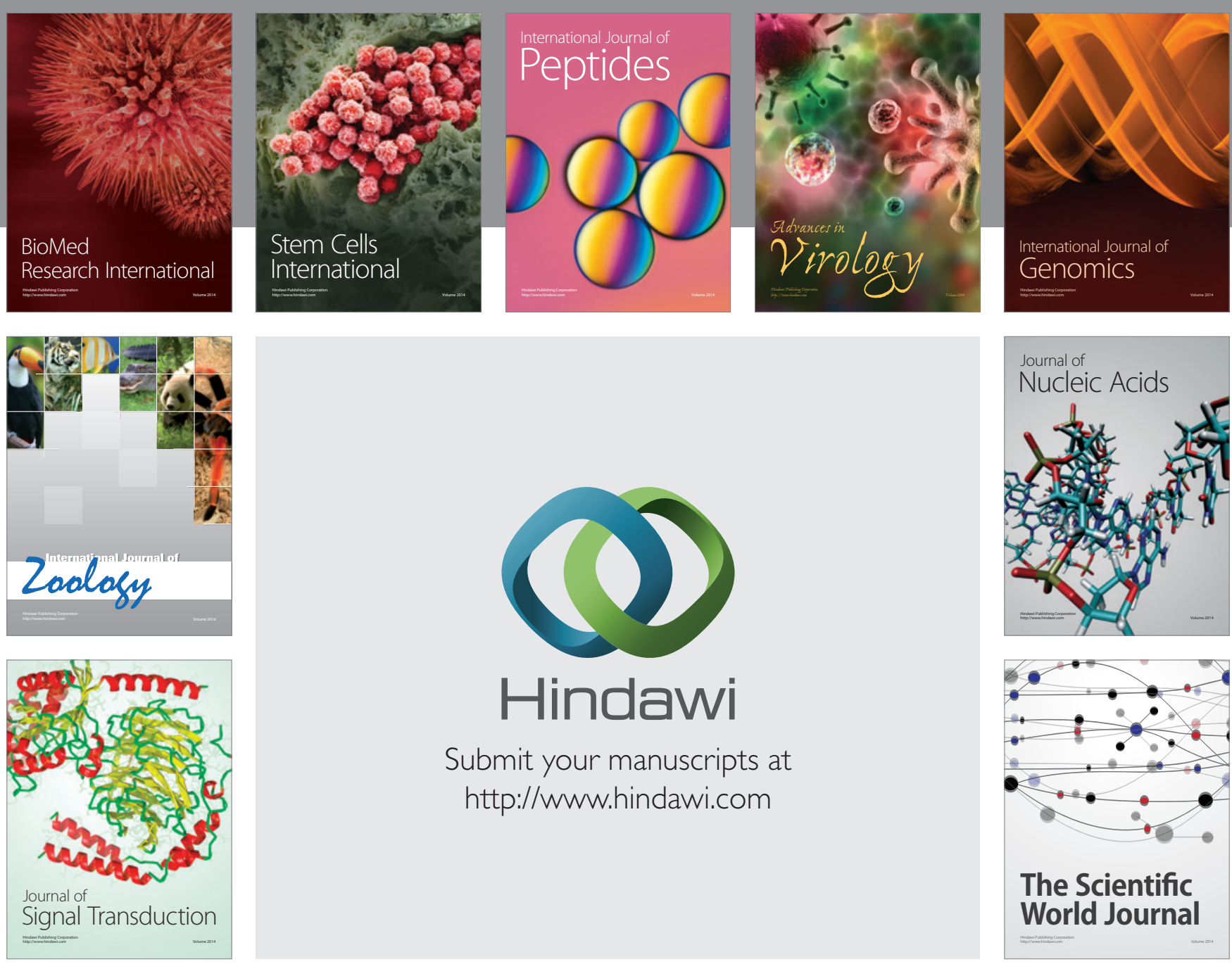

Submit your manuscripts at

http://www.hindawi.com
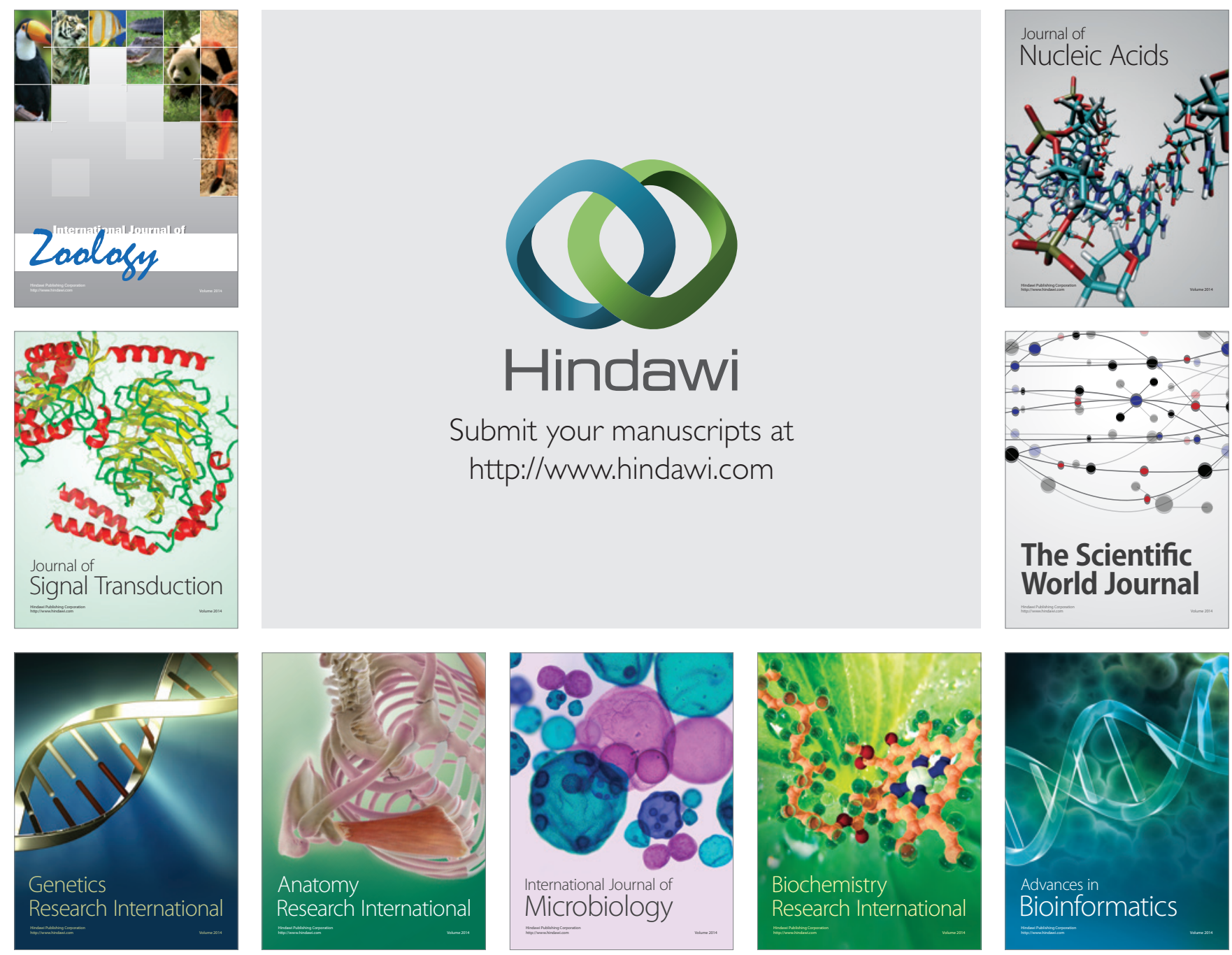

The Scientific World Journal
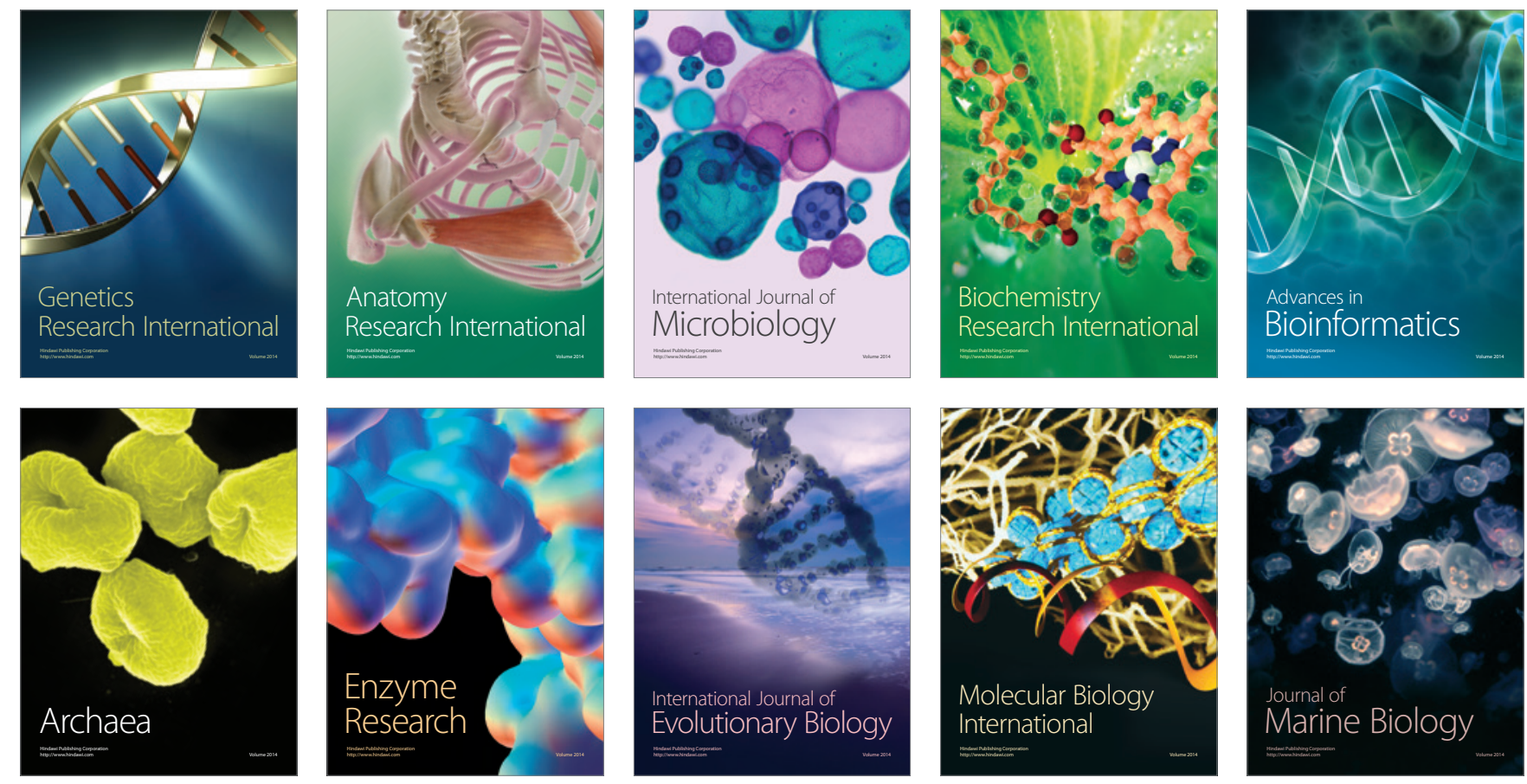